\title{
Mobile Health Applications for Rehabilitation of Musculoskeletal Diseases of the Shoulder: A Systematic Analysis
}

\author{
Bianca STEINER ${ }^{\mathrm{a}, 1}$, Isabel RICHTER ${ }^{\mathrm{a}}$, Lena ELGERT ${ }^{\mathrm{b}}$, \\ Reinhold HAUX ${ }^{\mathrm{a}}$ and Klaus-Hendrik WOLF $^{\mathrm{b}}$ \\ ${ }^{a}$ Peter L. Reichertz Institute for Medical Informatics of TU Braunschweig and \\ Hannover Medical School, Braunschweig, German \\ ${ }^{\mathrm{b}}$ Peter L. Reichertz Institute for Medical Informatics of TU Braunschweig and \\ Hannover Medical School, Hannover, Germany
}

\begin{abstract}
Rehabilitation of musculoskeletal diseases (MSD) of the shoulder is a multifaceted long-term process, which is often not transparent to affected patients. Mobile health applications (apps) have the potential to support this complex process by improving patients' self-management skills. However, there seems to be a lack of apps providing a holistic approach to motivate and guide patients during the whole rehabilitation process. Therefore, a systematic analysis of apps on Google Play Store was conducted by two independent reviewers. A total of 3227 apps were identified, of which 64 met the eligibility criteria for the qualitative analysis. The majority of analyzed apps were developed generally for patients with MSD of the shoulder, rarely for specific diseases (individual needs of patients). The majority of apps focus on the provision of information, exercise training, and alternative medicine. Apps for diagnostics, inpatient treatment, and self-management, especially for multiple rehabilitation phases, are rare or even not existent. Game design elements are seldom used. If there are any, then simple to implement ones, e.g. messages and progress bars. The (psychological) effects of individual game design elements on patients seem to be neglected, when selecting and implementing game-components.
\end{abstract}

Keywords. Musculoskeletal Diseases, Shoulder, Rehabilitation, Mobile Applications, Gamification, Disease Management, Self-Management

\section{Introduction}

Rehabilitation of musculoskeletal diseases (MSD) of the shoulder includes much more than only medical rehabilitation. Starting with primary and continuative diagnostics of MSDs of the shoulder, such as frozen shoulder or shoulder impingement syndrome (SIS), and proceeding with acute treatment to non-surgical or surgical treatment, rehabilitation ranges from inpatient and/or outpatient orthopedic rehabilitation up to subsequent rehabilitation services [1]. The conservative treatment, includes measures, such as exercise therapy, physical therapy, and pain therapy complemented by

\footnotetext{
${ }^{1}$ Corresponding Author, Bianca Steiner, Peter L. Reichertz Institute for Medical Informatics of TU Braunschweig and Hannover Medical School, Mühlenpfordtstr. 23, 38106 Braunschweig, Germany; E-mail: bianca.steiner@plri.de
} 
concomitant treatments, for example naturopathy, alternative medicine, and homeremedies [2,3]. Another important component of rehabilitation is prevention. In addition to exercises designed specifically for shoulder joints, this also includes general sports, fitness and activities, like swimming, Yoga, or Pilates. This multitude of services results in very complex individual rehabilitation processes (patient pathway), which are often not transparent to affected patients. Mobile health applications (apps) have the potential to support such complex processes by improving patients' selfmanagement skills [4,5]. Although some apps are already known for self-management in rehabilitation of chronic diseases, like diabetes, lower back pain, or asthma, such apps are rather rare for MSDs of the shoulder [4,5,6]. Overall, there seems to be a lack of apps, or application systems in general, providing holistic approaches to motivate and guide patients during the entire rehabilitation process - prevention, inpatient/outpatient rehabilitation, and aftercare. But especially for chronic diseases, adherence is a crucial factor for sustainable rehabilitation [7]. Gamification has emerged as a promising concept to increase motivation and adherence in therapeutic areas [8]. However, the extent to which game design elements are already finding their way into the growing mHealth app market, remains unclear.

The aim of this work is to provide an overview of apps to support rehabilitation of patients with MSDs of the shoulder. Here, the focus is on the fields of application and used game design elements, meaning game-components, game-mechanics, and gamedynamics. By analyzing existing fields of application and those in which the potentials for support has not yet been exhausted, gaps are to be detected. This way, starting points for new, holistic and motivating rehabilitation apps should be identified.

\section{Methods}

Following the PRISMA extension for scoping reviews (PRISMA-ScR), a systematic analysis of apps for rehabilitation of MSDs of the shoulder was performed on Google Play Store, the largest and most frequently used app store in Germany [9,10].

\subsection{Inclusion and Exclusion Criteria}

In principle, every app focusing on the treatment or management of patients with MSDs of the shoulder was included. According to ICD-10 this covers the following disorders: fractures, luxation, inflammatory diseases, and degenerative diseases. Apps focusing on sports and fitness rather than health were excluded. Therefore, it was examined whether a direct association with rehabilitation is discernible. Apps that are no longer available, not downloadable, not functioning, or not usable without a patient code were also excluded. Overall, apps implemented in languages other than English or German were translated if possible, otherwise excluded.

\subsection{Search Strategy}

Searching was done through the web interface of Google Play Store using 17 phrases, built up from the combination of the term 'shoulder' with the following nine keywords related to rehabilitation processes: rehabilitation, reha, therapy, prevention, pain management, self-management, pathway, guideline, and disease management. Each search was conducted in German and English. 
After removing duplicates, an initial screening of app descriptions and screenshots was done by two independent reviewers (BS, IR) according to the pre-defined inclusion and exclusion criteria. A third reviewer (LE) was consulted for final discussion in case of any discrepancies. All apps were assigned either to the category included, excluded, or applicable. Applicable apps are those not explicitly developed for the treatment and/or support of patients with MSDs of the shoulder, but which could certainly be suitable, as they are intended for orthopedic disorders, unspecified shoulder injuries, or general shoulder pain. As far as possible, all included apps were downloaded and assessed for eligibility by evaluating the apps' functionalities via the Android emulator 'BlueStacks' [11]. Once again, a classification into one of the three categories was used.

\subsection{Synthesis of Results}

The analysis is based on descriptive, qualitative and content-related aspects. Quality of apps was assessed by comparing user ratings in the store with the results of the 'Mobile App Rating Scale' (MARS) [12]. MARS is an easy-to-use, objective and reliable questionnaire for assessing an app's quality in five dimensions: (1) engagement, (2) functionality, (3) aesthetics, (4) information quality, and (5) subjective quality [12]. All apps are tested for at least 5 minutes and evaluated by two reviewers (BS, IR). For MARS-scoring, the mean of each 23 questions is used. The additional content analysis is based on a deductively developed code tree [13], consisting of three main categories: population, scope, and game design elements. Whenever necessary, the sub-categories already determined are supplemented by subsumption (inductively categorization).

\section{Results}

The search was conducted from January 17 to 23, 2020 and resulted in 3227 matches (see Fig. 1). After removing duplicates and initial app-screening, 113 apps were downloaded and included for app-screening. Thereby, 64 apps met the eligibility criteria for the qualitative analysis. 72 additional apps were categorized as applicable, because although they are potentially useful for shoulder rehabilitation, they do not contain specific functionalities for MSDs (individual needs of patients).

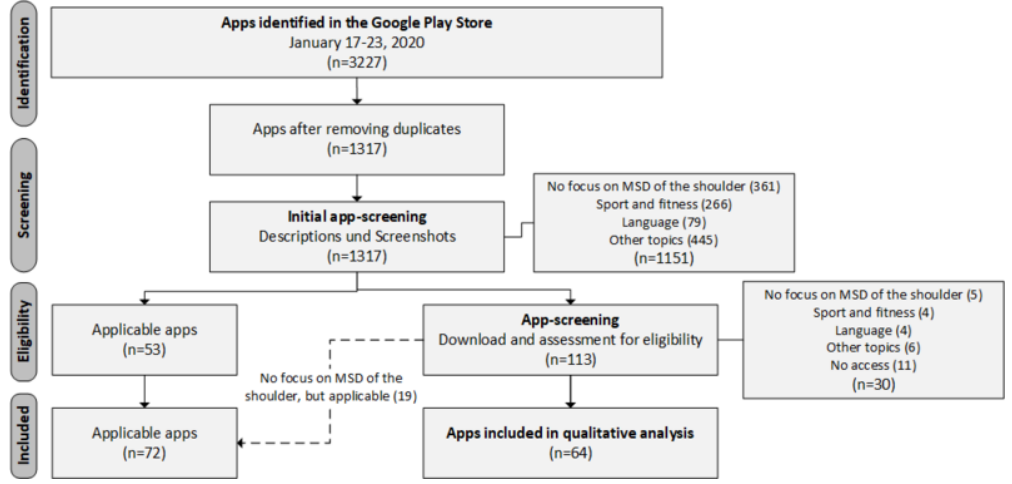

Figure 1: Screening process of the search in Google Play Store in January 2020 


\subsection{App Characteristics and Quality Rating}

There are 26 of the 64 apps available on Apple and Android devices. Thereby, the majority are accessible free of charge. For four apps there are one-time download costs, ranging from $1.09 €$ to $7.99 €$ [14-17]. For five additional apps, regular costs of between $8.99 €$ and $14.99 €$ per month are incurred through subscriptions [18-22]. Compared to most free apps, however, these receive updates more frequently. The number of downloads varies. While four apps were downloaded less than 10 times [16,23-25], 12 apps were downloaded more than 10.000 times [19,20,26-35], the app 'Shoulder, neck pain relief' [27] even more than 50.000 times.

More than half of all apps are not rated $(n=33)$. Just 12 apps were rated more than 50 times [18-20,27-31,34-36], only four of them more than 100 times [28-31]. The most frequently rated app is 'Doctor Homeopathy' [30] with 221 votes. On average, apps were rated with a score of $4.2( \pm 0.47)$ points. The three best apps, with 4.8 points, are 'Shoulder Rehabilitation Exercises' [26], 'Divine Care: Acupressure Points, Health \& Beauty' [37], and 'iShoulder' [38]. However, MARS does not reflect this valuation. Here, the best rated apps are 'Vivira - Physiotherapy Exercises at Home' [20], 'Rheuma Auszeit' [32], and 'Ouchie: your pain management companion' [36]. Overall, only ten apps with a MARS-score $>4$ (max. score $=5)$ could be considered as good. Generally, apps were rated less favorable with an average score of $3.31( \pm 1.45)$ points. Table 1 shows the average ratings for each MARS-dimension.

Table 1. App ratings per MARS-dimension (*best rated apps will only be named in case of a unique rating)

\begin{tabular}{lccc}
\hline Dimension & Average score & Standard deviation & Best rated app* \\
\hline Engagement & 2.42 & 1.00 & Vivira (4.6) \\
\hline Functionality & 4.11 & 0.74 & 6 with max. score \\
\hline Aesthetics & 3.53 & 0.46 & 5 with max. score \\
\hline Information quality & 3.20 & 0.97 & $\begin{array}{c}\text { Pathways Pain } \\
\text { Relief (4.83) }\end{array}$ \\
\hline Subjective quality & 2.37 & 0.66 & Vivira (4.74) \\
\hline
\end{tabular}

\subsection{Field of Application}

Most apps were developed generally for patients with MSDs of the shoulder $(n=30)$. Conversely, only few apps have been designed for specific shoulder diseases. There are single apps for SIS [35], fractures [39,43], and rotator cuff tears [19]. The most frequently addressed conditions are frozen shoulder $(n=14)$ and types of arthritis $(n=6)$.

The majority of apps is used to provide information $(n=19)$, for example on diagnostics, physical therapy, or alternative medicine. There are also numerous apps for performing exercises $(\mathrm{n}=16)$, practicing alternative medicine and home-remedies $(n=12)$, or managing pain $(n=8)$. Apps often integrate a kind of assessment tool for selfreflection $(n=13)$. However, functionalities for monitoring pathogenesis or therapy adherence are often unconsidered $(n=5)$. Prevention, diagnostics, and acute treatment are also rarely addressed fields of application. Merely four apps deal with selfmanagement of diseases [24,44-46]. Thereby, the apps 'Healo' [45] and 'Musculoskeletal (MSK) Self-Care' [46] seem particularly promising, in consideration of user ratings, MARS-score and implemented functionalities. For instance, 'Healo' 
offers customized self-care programs based on self-diagnosis for patients with rotator cuff tear, SIS, frozen shoulder, and shoulder instability [45]. Using the app as a digital therapeutic, patients are able to receive individual video exercise programs (therapy), rate their pain (assessment), answer questions about their state of health (diagnostics), and track their performance in real-time (monitoring) [45]. Nevertheless, even 'Healo' does not offer support for the entire rehabilitation process.

\subsection{Gamification}

Just 12 apps make use of game design elements ${ }^{2}$. Thereby, the most commonly used game-components are messages/information $(\mathrm{n}=7)$, tasks $(\mathrm{n}=6)$, progress bars $(\mathrm{n}=5)$, and social media $(\mathrm{n}=5)$. Most often, these components are employed to realize the game-mechanics feedback $(\mathrm{n}=7)$, cooperation $(\mathrm{n}=4)$, and education $(\mathrm{n}=2)$. Ultimately, this is intended to address the game-dynamics progress $(n=6)$, relationship $(n=5)$, and in parts also emotions $(n=3)$. For example, the app 'Limber Health' [24] uses the game-components task, progress bars, and achievements in the management of musculoskeletal injuries to provide feedback, generate winning conditions, and challenges. This way the game-dynamics emotions and progress are addressed to increase interactivity and enjoyment during exercise training, and thus finally have a motivating effect.

Overall, those five apps implementing more than three game-components scored particularly well in MARS (4.21 \pm 0.3$)$. 'Pathways Pain Relief' [18] is one of these well rated apps (user ratings $=4.1$; MARS-score $=4.26$ ). The app is designed for pain management in frozen shoulder patients. By completing tasks, new content can be gradually unlocked. Feedback is provided via audio content and progress bars. Furthermore, the app offers possibilities to interact with other affected users via integrated social media content.

Astonishingly, easy to implement game-components, like points $(\mathrm{n}=1)$ and achievements $(\mathrm{n}=2)$, are rarely utilized. Levels, rankings, and teams are not used at all. This applies to game-mechanics as well. While challenges $(\mathrm{n}=1)$ and winning conditions $(\mathrm{n}=2)$ are rarely used, e.g. rewards and transactions are not realized at all. Thus, it can be stated that the potential of gamification to increase motivation and adherence is seldom exploited.

\section{Discussion}

The aim of this work was to identify apps supporting patients with MSDs of the shoulder during rehabilitation, and to analyze them in terms of the fields of application and used game design elements. In total, 64 apps focusing on shoulder rehabilitation could be identified in the Google Play Store. Most of these apps deal with exercise training. Apps to assist patients during the entire rehabilitation process do not seem to exist. Furthermore, the minority of apps utilize game design elements for additional motivation of patients.

The market situation of rehabilitation apps for MSDs of the shoulder was analyzed only in Google Play Store. An additional search in the Apple App Store was refrained

${ }^{2}[16,18,20,22,25,33-35,37-40]$ 
from. Given the market share of Android in Germany (75.8\%), it can be assumed that this additional search only brings rather little benefit relative to the effort [47].

Apps focusing on sports and fitness rather than health are excluded, despite being theoretically useful for prevention. This exclusion was necessary to avoid bias, since only apps specifically implemented for rehabilitation should be analyzed, as these are hopefully targeted to the needs of patients. For this reason, also the category of applicable apps was introduced. These apps are potentially useful for patients with MSDs of the shoulder, but not specifically designed for them. A supplementary analysis, not carried out so far, could be useful for these apps as well, to completely assess the current market situation and the potentials of apps for rehabilitation.

The rating of apps was significantly better by users than by MARS. This might be explained by the fact that with MARS all 64 apps were rated, whereas user ratings are only available for about half of the apps. This is also reflected in the relatively high standard deviation of 1.45 points in MARS. The MARS-rating was carried out only by two researchers. For a more comprehensive qualitative statement it would be interesting to have the apps evaluated by affected patients as well. Nevertheless, the approach applied seems to be sufficient for a first qualitative assessment, as also other similar studies show $[48,49]$.

\section{Conclusion}

In general, there is a variety of sports and fitness apps on the Google Play Store. Tailored for rehabilitation of MSDs of the shoulder there are only a few apps available. Most of these apps focus on a pure provision of information, exercise training, or alternative medicine. Apps for diagnostics, inpatient treatment, and self-management are rare. Disease management apps for multiple rehabilitation phases seem to exist only for other chronic diseases. Such self-care apps, like 'Woebot' [50], 'RHD Treatment Tracker' [51], and 'eQuoo' [52] employ game design elements for behavior changes and motivation. Therefore, it is striking that despite these positive effects of gamification, with the exception of messages/information and simple tasks, game design elements are rarely used in apps for rehabilitation of MSDs of the shoulder. This solely indicates the potentials for short-term motivation. The capabilities of selfmanagement apps to cleverly accompany the entire rehabilitation process of patients with MSD of the shoulder, both in short- and long-term, can only be guessed at.

\section{Conflict of Interest}

The authors state that they have no conflict of interests.

\section{References}

[1] R.E. Holmes, W.R. Barfield, and S.K. Woolf, Clinical evaluation of nonarthritic shoulder pain: Diagnosis and Treatment, Phys Sportsmed 43 (2015), 262-268.

[2] B. Greitemann, and V. Stein, Praxis der konservativen Orthopädie - Rehabilitation in der Orthopädie, Orthopädie und Unfallchirurgie 8 (2013), 503-522.

[3] L. Chaitow, Naturopathic Physical Medicine: Theory and Practice for Manual Therapists and Naturopaths, Elsevier Health Sciences, Edinburgh, 2008. 
[4] G.M. Coorey, L. Neubeck, J. Mulley, and J. Redfern, Effectiveness, acceptability and usefulness of mobile applications for cardiovascular disease self-management: Systematic review with metasynthesis of quantitative and qualitative data, Eur J Prev Cardiol 25 (2018), 505-521.

[5] G.C. Machado, M.B. Pinheiro, H. Lee, O.H. Ahmed, P. Hendrick, C. Williams, and S.J. Kamper, Smartphone apps for the self-management of low back pain: A systematic review, Best Pract Res Clin Rheumatol 30 (2016), 1098-1109.

[6] R. Nussbaum, C. Kelly, E. Quinby, A. Mac, B. Parmanto, and B.E. Dicianno, Systematic Review of Mobile Health Applications in Rehabilitation, Arch Phys Med Rehabil 100 (2019), 115-127.

[7] J. Dunbar-Jacob, J.A. Erlen, C.M. Ryan, S.M. Sereika, and W.M. Doswell, Adherence in chronic disease, Annu Rev Nurs Res 18 (2000), 48-90.

[8] D. Johnson, S. Deterding, K-A. Kuhn, A. Staneva, S. Stoyanov, and L. Hides, Gamification for health and wellbeing: A systematic review of the literature, Internet Interventions 6 (2016), 89-106.

[9] A.C. Tricco, E. Lillie, W. Zarin, K.K. O'Brien, H. Colquhoun, D. Levac, et al. PRISMA Extension for Scoping Reviews (PRISMA-ScR): Checklist and Explanation, Ann Intern Med 168 (2018), 567-473.

[10] Statista, Number of apps available in leading app stores as of 4th quarter 2019, 2020. https://www.statista.com/statistics/276623/number-of-apps-available-in-leading-app-stores/ (accessed Jan 14, 2020).

[11] BlueStacks, BlueStacks - Not Another Android Emulator, 2020. https://www.bluestacks.com/ (accessed Feb 22, 2020).

[12] S.R. Stoyanov, L. Hides, D.J. Kavanagh, O. Zelenko, D. Tjondronegoro, and M. Mani, Mobile app rating scale: a new tool for assessing the quality of health mobile apps, JMIR Mhealth Uhealth 3 (2015).

[13] J. Forman, and L. Damschroder, Qualitative Content Analysis, in: L. Jacoby, L. Siminoff, Empirical Methods for Bioethics: A Primer, Emerald Publishing Limited, Bingley, 2007, 39-62.

[14] Urodev, iTraumApp Shoulder Pro, v1.0.3, 2014. https://play.google.com/store/apps/details?id=com. ACSimulation.iTraumaHombroPro (accessed Jan 17, 2020).

[15] Center for Pain and Stress Research, MPS Shoulder Therapy, v1.0, 2019. https://play.google.com/store/apps/details?id=com.dolphin.mps (accessed Jan 17, 2020).

[16] Colin McDonnell, Orthopedic Referral Guidelines, v1.0, 2017. https://play.google.com/store/apps/ details?id=com.cleverstagortho (accessed Jan 17, 2020).

[17] PhysioAdvisor, PhysioAdvisor Exercises, v1.4, 2013. https://play.google.com/store/apps/details?id= com.appiphany.physio (accessed Jan 17, 2020).

[18] Natural, proven \& safe chronic pain relief, Pathways Pain Relief, v2.7.3, 2019. https://play.google.com/store/apps/details?id=com.pathways.Pathwayspainrelief (accessed Jan 7, 2020).

[19] Injurymap, Injurymap - Effective exercise therapy, v1.5.42, 2020. https://play.google.com/store/apps/ details?id=com.injurymap.injurymap (accessed Jan 17, 2020).

[20] Vivira Health Lab GmbH, Vivira - Physiotherapy Exercises at Home, v2.15.0, 2020. https://play.google.com/store/apps/details?id=com.vivira.android (accessed Jan 17, 2020).

[21] 9zest, 9zest FixHealth - Pain, Stress, 2018. https://play.google.com/store/apps/details?id=com. ninezest.fixhealth (accessed Jan 17, 2020).

[22] Mehab, LLC, MEHAB - Mobile Physical Therapy, v2.3, 2020. https://play.google.com/store/apps/ details?id=com.application.mehab (accessed Jan 17, 2020).

[23] Medics-paramedicsapp, Orthopedics Shoulder \& Elbow, v2.0, 2019. https://play.google.com/store/ apps/details?id=com.orthopedicupperextremity.medical apps (accessed Jan 17, 2020).

[24] Limber Health, Limber Health, v1.0, 2020. https://play.google.com/store/apps/details?id=com. limberhealth.mobileapp (accessed Jan 17, 2020).

[25] aDRp, Needleless Acupuncture: Self Treatment Guide, v1.0, 2019. https://play.google.com/store/apps/ details?id=com.NeedlelessAcupuncture (accessed Jan 17, 2020).

[26] Sharudin, Shoulder Rehabilitation Exercises, v1.2, 2019. https://play.google.com/store/apps/ details?id=net.jombalik.shoulderehabexercise (accessed Jan 17, 2020).

[27] Fitness Lab, Shoulder, neck pain relief, v2.5, 2018. https://play.google.com/store/apps/ details?id=com.workoutapps.shoulder.stretching.exercises (accessed Jan 17, 2020).

[28] Healure Technology, Healure: Physiotherapy Exercise Plans, v1.1.07, 2019. https://play.google.com/store/apps/details?id=com.healure.painrecovery (accessed Jan 17, 2020).

[29] Fadevs, Clinical Orthopaedics Surgery, v2.0, 2019. https://play.google.com/store/apps/ details?id=com.andromo.dev710410.app830149 (accessed Jan 17, 2020).

[30] VTSBRO Apps, Doctor Homeopathy, v1.3, 2019. https://play.google.com/store/apps/ details?id=com.zddapps.homeophatic (accessed Jan 17, 2020).

[31] Nubia Suarez, Remedios Caseros Artritis, v5.0, 2019. https://play.google.com/store/apps/ details?id=com.andromo.dev605364.app627733 (accessed Jan 17, 2020).

[32] Deutsche Rheuma-Liga Bundesverband e.V., Rheuma-Auszeit, v1.0, 2019. https://play.google.com/ store/apps/details?id=com.mobile.rheumaauszeit (accessed Jan 17, 2020). 
[33] Health \& Fitness Guide, Home Remedies and Natural Cure, v1.0, 2017. https://play.google.com/store/apps/details?id=com.HealthAndFitnessGuide.HomeRemediesAndNatural Cure (accessed Jan 17, 2020).

[34] Multi App Logix, Physiotherapy Exercises by Dr. Huma Ibrar Abbasi, v1.0, 2017. https://play.google.com/store/apps/details?id=com.multiapplogix.physiotherapytips (accessed Jan 17, 2020).

[35] Physera, Physera, v5.0.2, 2020. https://play.google.com/store/apps/details?id=com.healthcoda.physera (accessed Jan 17, 2020).

[36] Ouchie, Ouchie: your pain management companion, v1.5.11, 2020. https://play.google.com/store/apps/ details?id=com.ouchie (accessed Jan 17, 2020).

[37] Divine Care, Divine Care: Acupressure Points, Health \& Beauty, v1.0, 2020. https://play.google.com/store/apps/details?id=com.siddharth.divinecareapp (accessed Jan 17, 2020).

[38] Alberto Sanchez, iShoulder, v1.0, 2013. https://play.google.com/store/apps/details?id=com. albertosanchezmac.myshoulder (accessed Jan 17, 2020).

[39] Grupo de Telemática e Imagen, Fisioterapia fractura hombre, v1.0, 2018. https://play.google.com/store/ apps/details?id=com.gti.maria.physiotherapyapp (accessed Jan 17, 2020).

[40] MultimediaTechnology, ShoulderApp, v1.2, 2016. https://play.google.com/store/apps/details?id=com. fachhochschulesalzburggmbh.ShoulderApp (accessed Jan 17, 2020).

[41] The Painspace, the painspace: for pain relief, v1.1.21, 2018. https://play.google.com/store/apps/ details?id=com.thepainspace.ThePainspace (accessed Jan 17, 2020).

[42] Eva-Maria Zettl, Der Sportdoktor, v6.030, 2019. https://play.google.com/store/apps/details?id=com. Tobit.android.Slitte7550914094 (accessed Jan 17, 2020).

[43] HindiTreading Apps, Neck, shoulder pain relief, v2.0, 2019. https://play.google.com/store/apps/ details?id=com.hindi_treadinapps.shoulder_neck_pain (accessed Jan 17, 2020).

[44] PHYSIO PLUS, Prakruti Physiotherapy Clinic, v0.0.1, 2019. https://play.google.com/store/apps/ details?id=com.physioplusnetwork.prakruti (accessed Jan 17, 2020).

[45] Empowered Applications, Healo, v1.2.5, 2020. https://play.google.com/store/apps/details?id=com. empowered.healo (accessed Jan 17, 2020).

[46] NHS Leicestershire Health Informatics Service, Musculoskeletal (MSK) Self-Care, v1.0, 2019. https://play.google.com/store/apps/details?id=uk.nhs.leics.his.msk (accessed Jan 17, 2020).

[47] Statista, Market share comparison of Android and iOS in smartphone sales in Germany from January 2012 to December 2019, 2020. https:/www.statista.com/statistics/461900/android-vs-ios-market-sharein-smartphone-sales-germany (accessed Feb 13, 2020).

[48] J. Knitza, K. Tascilar, E.M. Messner, M. Meyer, D. Vossen, A. Pulla, et al. German Mobile Apps in Rheumatology: Review and Analysis Using the Mobile Application Rating Scale (MARS), JMIR Mhealth Uhealth 8 (2019), e14991.

[49] H. Jamaladin, T.H. van de Belt, L.C. Luijpers, F.R. de Graaff, S.J. Bredie, N. Roeleveld N, et al. Mobile Apps for Blood Pressure Monitoring: Systematic Search in App Stores and Content Analysis, JMIR Mhealth Uhealth 11 (2018),e187.

[50] Woebot Labs, Woebot: Your Self-Care Expert, v3.16.4, 2020. https://play.google.com/store/apps/ details?id=com.woebot (accessed Apr 01, 2020).

[51] RHDAustralia, RHD Treatment Tracker, v1.0.5, 2019. https://play.google.com/store/apps/ details?id=au.edu.menzies.rhdtreatmentracker2 (accessed Apr 01, 2020).

[52] PsycApps Ltd. (UK), eQuoo: Das Emotionale Fitness Spiel, v3.3.2, 2019. https://play.google.com/store/apps/details?id=com.bitbox.equoo (accessed Apr 01, 2020). 